\title{
An Analysis of Pulsed Wave Ultrasound Systems for Blood Velocity Estimation
}

Jensen, J. A.

Published in:

Acoustical Imaging

Publication date:

1995

Document Version

Peer reviewed version

Link back to DTU Orbit

Citation (APA):

Jensen, J. A. (1995). An Analysis of Pulsed Wave Ultrasound Systems for Blood Velocity Estimation. Acoustical Imaging, 22, 377-384.

\section{General rights}

Copyright and moral rights for the publications made accessible in the public portal are retained by the authors and/or other copyright owners and it is a condition of accessing publications that users recognise and abide by the legal requirements associated with these rights.

- Users may download and print one copy of any publication from the public portal for the purpose of private study or research.

- You may not further distribute the material or use it for any profit-making activity or commercial gain

- You may freely distribute the URL identifying the publication in the public portal

If you believe that this document breaches copyright please contact us providing details, and we will remove access to the work immediately and investigate your claim 
Paper presented at Acoustical Imaging 22, 1995:

\title{
AN ANALYSIS OF PULSED WAVE ULTRASOUND SYSTEMS FOR BLOOD VELOCITY ESTIMATION
}

\author{
Jørgen Arendt Jensen, \\ Electronics Institute, Build. 349, \\ Technical University of Denmark, \\ 2800 Lyngby, Denmark
}

Published in Acoustical Imaging 22, pp. 377-384, 1995. 


\title{
AN ANALYSIS OF PULSED WAVE ULTRASOUND SYSTEMS FOR BLOOD VELOCITY ESTIMATION
}

\author{
Jørgen Arendt Jensen \\ Electronics Institute, Build. 349, \\ Technical University of Denmark, \\ 2800 Lyngby, Denmark
}

\begin{abstract}
Pulsed wave ultrasound systems can be used for determining blood's velocity non-invasively in the body. A region of interest is selected, and the received signal is range gated to measure data from the region. One complex sample value is acquired for each pulse emission after complex demodulation of the received signal. The time evolution and distribution of velocity can then be found by using samples from a number of pulse-echo lines. Making a short-time Fourier transform of the data reveals the velocity distribution in the range gate over time.

Such systems are called Doppler ultrasound systems implying that they use the classical Doppler effect. The velocity is typically on the order of 0.5 to $1 \mathrm{~m} / \mathrm{s}$ giving a relative shift of 2 to $4 \mathrm{kHz}$ of the center frequency of the received spectrum for a $3 \mathrm{MHz}$ transducer. Finding such a shift is impossible since the unknown frequency shift from attenuation in tissue can be tens of kilohertz. Some recent reviews and articles state that the Doppler effect is used, and contradictory and wrong results and erroneous system diagrams arise from this assumption. Research done in the last fifteen years has revealed that it is the movement of the scatterers between pulse emissions, that is used for finding the velocity. This finding gives new insight into the role of the complex demodulation stage, and shows that this can be replaced by a matched filter and quadrature RF sampling. A derivation of this result is presented in this paper, and it reveals how the bandwidth of the pulse and the number of pulse emissions affect the result. The final equation for the received signal is quite complicated, and a simplified interpretation is therefore also given. This readily reveals the influence from transducer bandwidth, attenuation, non-linear effects, classical Doppler effect, and scattering.
\end{abstract}

\section{INTRODUCTION}

Pulsed ultrasound systems for the investigation of blood velocity emerged in the early $1970 \mathrm{~s}$. A seminal paper was that by Baker (1970), which detailed the sampling operation of the received backscattered signal from the blood. A single sample was acquired for each pulse emitted and Baker stated that the system tracked the slow movement of the scatterers through the range gate. Unfortunately Baker also calls this the Doppler effect, and the name has since then stuck to these systems, and has produced a number of mistakes in interpreting the function of and signal processing in these systems. It is here important to differentiate between the interaction between the moving scatterers and the pulse, yielding the Doppler shift in frequency, and the inter-pulse movement of the scatterers. The latter is the effect detected in these systems as shown in Section 2. A model for the inter-pulse movement is derived in Section 3 and used in Section 4 for calculating the spectrum of the received signal. It is shown that sampling can be performed at a frequency in the $\mathrm{kHz}$ range although the received signal is in the $\mathrm{MHz}$ range. The derived model is somewhat complicated, and a simpler interpretation using frequency scaling is given in Section 5. From this the influence of, e.g., attenuation and non-linear propagation is readily revealed and the role of the various parts of a PW system explained.

This paper shows that the frequency axis for the received signal is scaled by the factor $2 \mathrm{v} / \mathrm{c}$, where $c$ is speed of sound and $v$ is blood velocity. This time dilation was first explicitly noted 


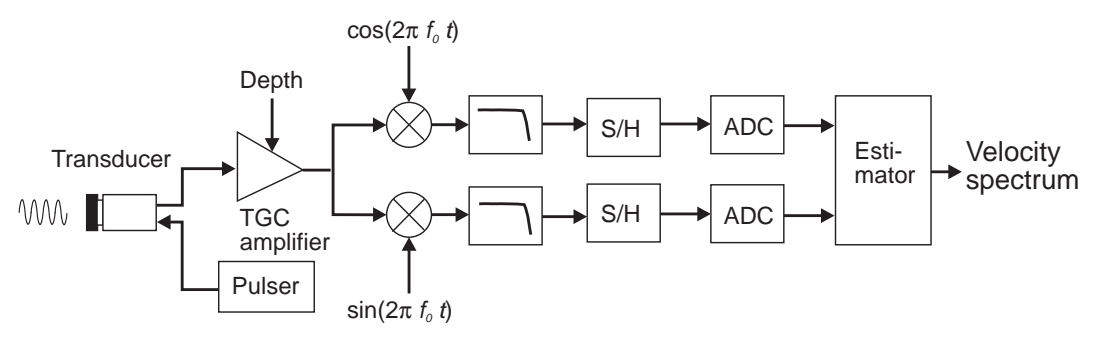

Figure 1: Block diagram of pulsed wave system for velocity estimation.

by Newhouse and Amir (1983). They gave a model for the received signal that demonstrated the stretched time factor. Experiments were also shown proving the model correct. The model was derived earlier and used for evaluating transit time effects (Newhouse et al. 1976) and the effects from scattering and absorption on the power density spectrum (Newhouse et al. 1977), although the time dilation effect was not noted explicitly. The influence from attenuation was measured by Holland et al. (1984) and found in agreement with the model. The scaling of the time axis was also used by Magnin (1986). He presented the signal processing fundamentals for the first commercial system to apply direct RF sampling of the received signal, thus skipping the analog demodulation scheme. The exact same scheme was presented by Forsberg and Jørgensen (1989). Magnin (1986) derived a model for the received signal using the time shift effect for a Gaussian pulse and infinitively many pulse emissions. The commercial implementation of this system is given by Halberg and Thiele (1986). Thomas and Leeman (1991) have also considered the time shift model and point out the difference between this and the Doppler effect, and elucidated the time scaling involved in forming the received signal. The difference was also experimentally demonstrated by Thomas and Leeman (1993).

This paper derives the received signal for a direct RF sampled signal and extends the model to include a limited number of pulses. It is also shown that the direct sampled system and the more conventional ones using analog demodulation actually are equivalent. The only difference is the implementation of the matched filter in the RF receiver.

\section{DOPPLER SYSTEM}

A traditional Doppler system is depicted in Fig. 1. A pulse is emitted and interacts with the moving blood. A Doppler shift of the pulse spectrum is introduced as

$$
f_{d}=-\frac{2|\vec{v}|}{c} \cos (\theta) f_{0}
$$

as a result of the blood velocity. Here $|\vec{v}| \cos \theta$ is the blood velocity along the direction of the ultrasound beam, $c$ is the speed of sound, and $f_{0}$ is the center frequency of the emitted pulse. The received signal is then quadrature demodulated. This is done by moving the spectrum down to around $f=0$ by multiplying the signal with $\exp \left(j 2 \pi f_{0} t\right)$ and then remove the sum component through a set of low-pass filters ${ }^{1}$. The resulting signal is sampled and processed to yield the Doppler shift, and thereby the blood velocity and its sign.

This standard explanation of the function of the Doppler system has problems when attenuation of the ultrasound field by the tissue is included. The Doppler frequency is typically on the order of a few $\mathrm{kHz}$, whereas the shift due to attenuation is considerably higher as can be seen from Fig. 2. The downshift in center frequency as a function of depth is depicted for a $3 \mathrm{MHz}$ Gaussian pulse with a relative bandwidth of 0.1 . The unknown attenuation shift can easily be ten times the Doppler shift, even for moderate depths. It is, thus, not possible to

\footnotetext{
${ }^{1}$ These are often, falsely, perceived as being anti-aliasing filters before the sampling process. They actually act as matched filters. See Section 6.
} 


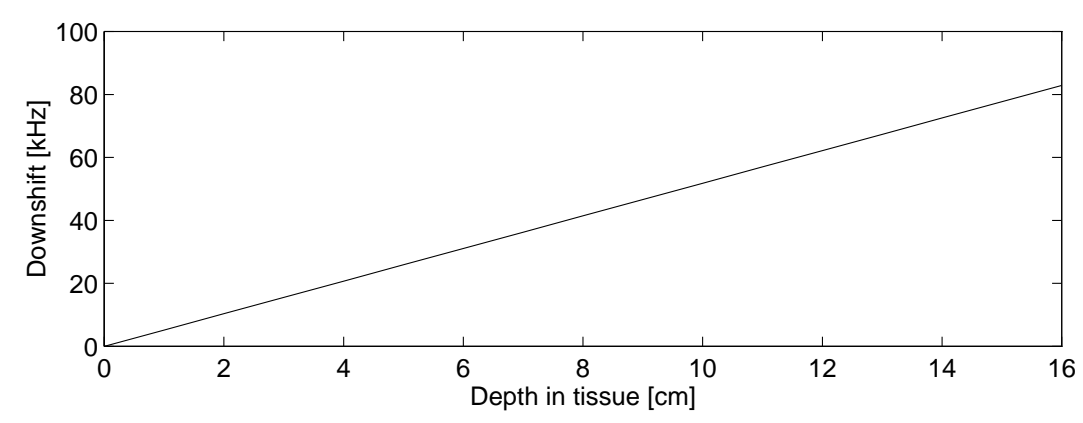

Figure 2: Shift in center frequency for a Gaussian pulse when propagating in tissue with an attenuation of $0.5 \mathrm{~dB} /[\mathrm{MHz} \mathrm{cm}]$. The center frequency is $3 \mathrm{MHz}$ and the relative bandwidth is 0.1 .

estimate the Doppler shift with any accuracy, and these systems do not employ the Doppler effect.

\section{TIME SHIFT MODEL}

A pulsed wave system for velocity estimation emits a number of pulses and extracts a single sample at the appropriate depth for each pulse. This sampled signal is then used for estimating the velocity or its spectrum. The estimation, thus, relies on the relation between samples in the sampled signal. This relation can be derived from the time shift of the signal from pulse to pulse. For a single scatterer $\vec{p}_{1}$ denotes the initial position of the scatterer and $\vec{p}_{2}$ the position after the next pulse emission $T_{\text {prf }}$ seconds later. The distance between the two positions is given by

$$
\vec{p}_{2}-\vec{p}_{1}=\vec{v} T_{p r f}
$$

The movement in the $z$-direction is perceived as a time shift in the received RF signal and is given by

$$
t_{s}=\frac{|\vec{v}| \cos (\theta)}{c / 2} T_{p r f}=\frac{2|\vec{v}| \cos (\theta)}{c} T_{p r f}=\frac{2 v_{z}}{c} T_{p r f} .
$$

Two received signals are related by:

$$
r_{2}\left(t_{r}\right)=r_{1}\left(t_{r}-t_{s}\right)
$$

when denoting time since pulse emission as $t_{r}$. Emitting a sinusoidal pulse, one received response is given by

$$
r_{r}\left(t_{r}\right)=a \sin \left(2 \pi f_{0}\left(t_{r}-\frac{2 d}{c}\right)\right)
$$

where $d$ is the initial depth of the scatterer. Receiving the response from a number of pulses gives

$$
r_{r}^{(i)}\left(t_{r}\right)=a \sin \left(2 \pi f_{0}\left(t_{r}-\frac{2 d}{c}-i t_{s}\right)\right)
$$

Taking one sample at a fixed time $t_{x}$ relative to pulse emission gives a sampled signal

$$
\begin{aligned}
r_{s}(i) & =-a \sin \left(2 \pi f_{0} \frac{2 v_{z}}{c} T_{p r f} i-\phi_{c}\right) \\
\phi_{c} & =2 \pi f_{0}\left(\frac{2 d}{c}-t_{x}\right)
\end{aligned}
$$

where $\phi_{c}$ is a fixed phase factor. The received and sampled signal, thus, has a frequency of

$$
f_{p}=-\frac{2|\vec{v}| \cos (\theta)}{c} f_{0}=-\frac{2 v_{z}}{c} f_{0}
$$

which has the same magnitude as the Doppler frequency, but is generated by the inter-pulse movement of the scatterers. 


\section{SPECTRUM OF RECEIVED SIGNAL}

The model derived in Section 3 assumed that the emitted and received signals are essentially monochromatic. In reality a short pulse (8-16 cycles) are employed, and that affects the spectrum of the received signal. The influence of the pulse shape and duration is analyzed in this section. The analysis is, with no loss of generality, done for an RF sampled system in which the demodulation is replaced by a matched filter (see Section 6).

RF samples for $N$ emitted pulses are received and the signal after the transducer is given by

$$
r_{s}(t)=a \sum_{i=0}^{N-1} e\left(t-i T_{p r f}-i t_{s}-\frac{2 d}{c}\right),
$$

where $t$ is absolute time since the first pulse emission $(i=0)$ and $e()$ is the signal emitted for each pulse. Transforming to the frequency domain gives:

$$
\begin{aligned}
R_{s}(f) & =a E(f) \sum_{i=0}^{N-1} \exp \left(-j 2 \pi f\left(i\left(T_{p r f}+t_{s}\right)+\frac{2 d}{c}\right)\right) \\
& =a E(f) \sum_{i=0}^{N-1}\left(\exp \left(-j 2 \pi f\left(T_{p r f}+t_{s}\right)\right)\right)^{i} \exp \left(-j 2 \pi f \frac{2 d}{c}\right) \\
& =a E(f) \frac{\sin \left(\pi f\left(T_{p r f}+t_{s}\right) N\right)}{\sin \left(\pi f\left(T_{p r f}+t_{s}\right)\right)} \exp \left(-j \pi f\left((N-1)\left(T_{p r f}+t_{s}\right)+\frac{4 d}{c}\right)\right)
\end{aligned}
$$

using that $\sum_{i=0}^{N-1} x^{i}=\frac{1-x^{N}}{1-x}$ and the Euler identities. This is the spectrum of the signal received by the transducer for a number of pulse emissions. An example is shown in Fig. 3 for a rectangular pulse with 8 sine periods at $3 \mathrm{MHz}$ and for 10 pulse-echo lines. The local maxima are at

$$
f=\frac{n}{T_{p r f}+t_{s}}=\frac{n f_{p r f}}{1+\frac{2 v_{z}}{c}} \approx n f_{p r f}\left(1-\frac{2 v_{z}}{c}\right)
$$

and their amplitudes are modified by the spectral amplitude of the pulse.

The received signal is then sampled with a frequency of $f_{p r f}=1 / T_{\text {prf }}$, which can be described by a multiplication with a series of $\delta$-functions as

$$
r_{f}(t)=r_{s}(t) \sum_{n=-\infty}^{\infty} \delta\left(t-n T_{p r f}\right) .
$$

The Fourier transform of this signal is

$$
\begin{aligned}
R_{f}(f) & =R_{s}(f) * \frac{1}{T_{p r f}} \sum_{m=-\infty}^{\infty} \delta\left(f-\frac{m}{T_{p r f}}\right) \\
& =\int_{-\infty}^{+\infty} \frac{1}{T_{p r f}} \sum_{m=-\infty}^{\infty} R_{s}(s) \delta\left(f-\frac{m}{T_{p r f}}-s\right) d s=\frac{1}{T_{p r f}} \sum_{m=-\infty}^{\infty} R_{s}\left(f-\frac{m}{T_{p r f}}\right) .
\end{aligned}
$$

Combining with the previous equation gives

$$
\begin{aligned}
R_{f}(f)=a f_{p r f} & \sum_{m=-\infty}^{\infty} E\left(f-m f_{p r f}\right) \frac{\sin \left(\pi\left(f-m f_{p r f}\right)\left(T_{p r f}+t_{s}\right) N\right)}{\sin \left(\pi\left(f-m f_{p r f}\right)\left(T_{p r f}+t_{s}\right)\right)} \\
& \times \exp \left(-j \pi\left(f-m f_{p r f}\right)\left((N-1)\left(T_{p r f}+t_{s}\right)+\frac{4 d}{c}\right)\right) .
\end{aligned}
$$

The sampling operation moves the components of the spectrum down to the range from $-f_{\text {prf }} / 2$ to $f_{\text {prf }} / 2$. The maximum of the pulse spectrum is at $f_{0}$. The sampling operation is essentially a modulus $f_{p r f}$ operation, so the maximum in the resulting spectrum is at

$$
f=n f_{p r f}\left(1-\frac{2 v_{z}}{c}\right) \bmod f_{p r f}=-\frac{2 v_{z}}{c} n f_{p r f}
$$



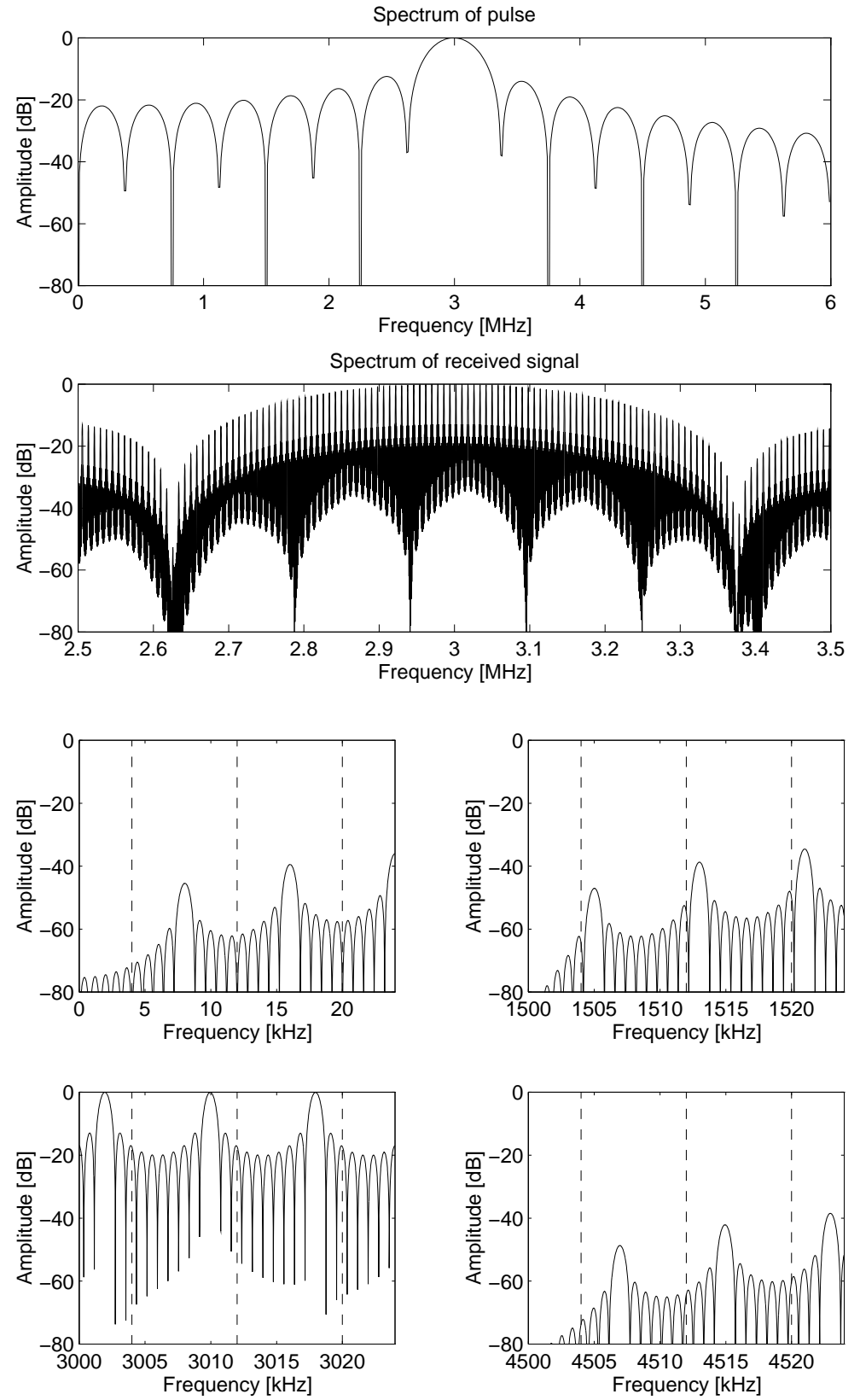

Figure 3: Spectrum of received signal at a velocity of $0.5 \mathrm{~m} / \mathrm{s} . M=8, N=10, f_{p r f}=8 \mathrm{kHz}$ (see Eq. (10)). The four small graphs are from different parts of the second graph.

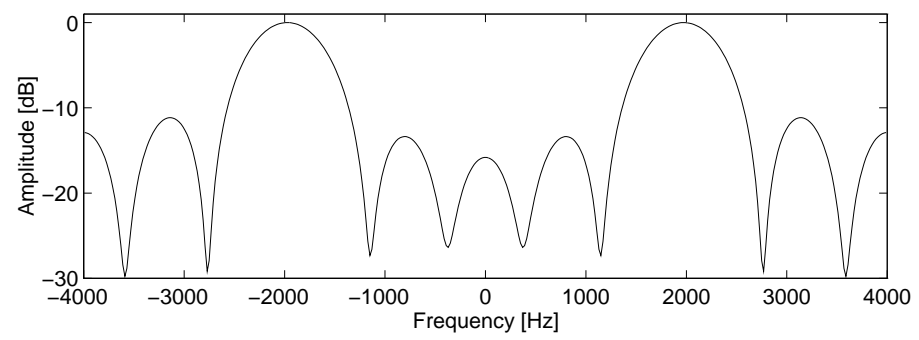

Figure 4: Resulting spectrum of received signal after sampling of signal with spectrum shown in Fig. 3. 

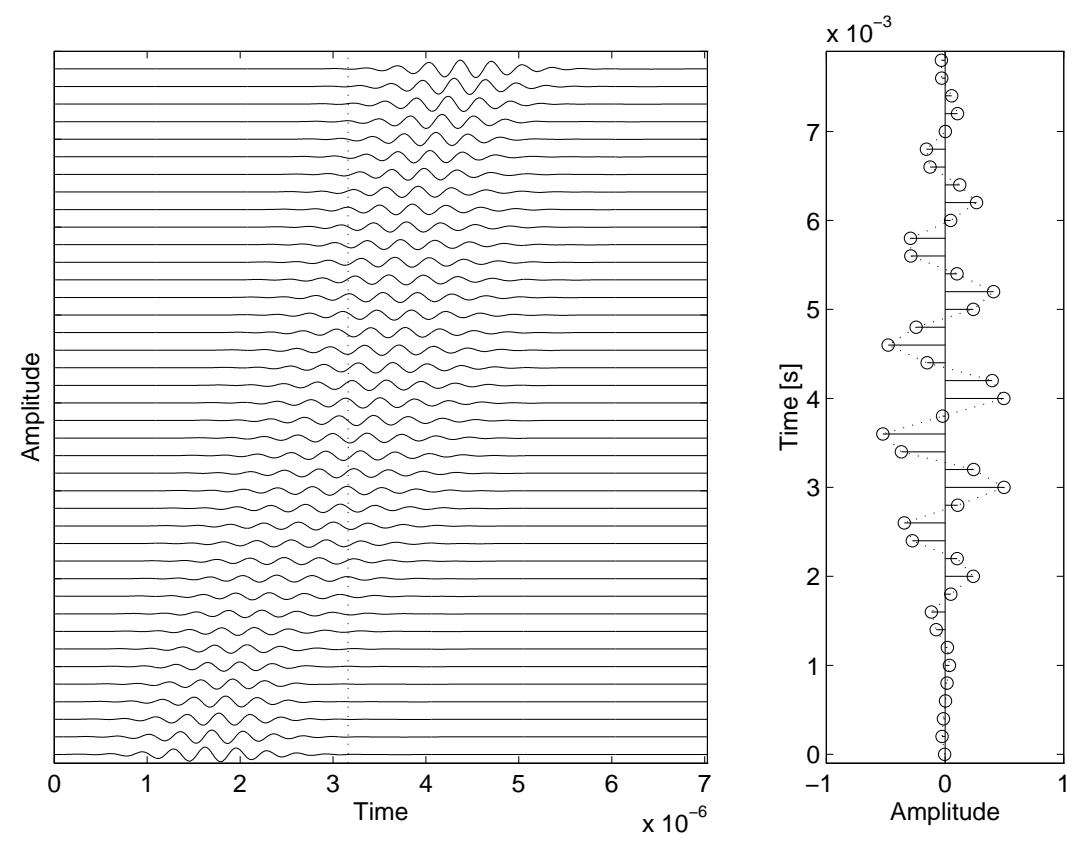

Figure 5: Signal from a single moving scatterer crossing a beam from a concave transducer.

and the dominant excursion is at $n f_{p r f} \approx f_{0}$, so the maximum is at

$$
f=-\frac{2 v_{z}}{c} f_{0}
$$

An example of this is seen in Fig. 4. The velocity is $0.5 \mathrm{~m} / \mathrm{s}$ and $f_{0}$ equals $3 \mathrm{MHz}$, giving a combined maximum at $f=\frac{2 \cdot 0.5}{1540} 3 \cdot 10^{6}=1948 \mathrm{~Hz}$. Two peaks are seen, since quadrature sampling is not employed.

\section{A SIMPLE INTERPRETATION}

Equation (14) reveals the effects from using different number of pulses and pulse shapes, but the consequence is not directly obvious from the equation. A simpler model would thus be beneficial. This can be derived by keeping in mind that the shift in position is responsible for generating the signal. An example is shown in Fig. 5. A single scatterer crosses the beam from a pulsed concave transducer. The resulting sampled signal has the same shape as the pulse, since the RF pulse slowly moves past the sampling point indicated by the dashed line. The consequence in the frequency domain can be seen from Eq. (7). All frequency components are multiplied with $2 v_{z} / c$, and are thus scaled to lie in the audio range. This scaling is indicated in Fig. 6. The RF frequency axis is replaced by the frequency axis for the sampled signal. The effect by a number of factors can now easily be deduced with this simple model in mind.

The pulse bandwidth is directly responsible for the bandwidth of the resulting spectrum. A longer pulse, thus, gives a more narrow spectrum. The influence from the number of pulseecho lines can also be deduced. Only part of the pulse is sampled if too few lines are used, and this amounts to multiplying a rectangular window onto the data. A smearing of the spectrum, thus, results. The sampling covers the full pulse length if

$$
\frac{2 v_{z}}{c} T_{p r f} N \geq \frac{M}{f_{0}}
$$

where $M$ is the number of sine periods in the pulse. The lowest velocity possible to determine is therefore dependent on the number of lines used. 


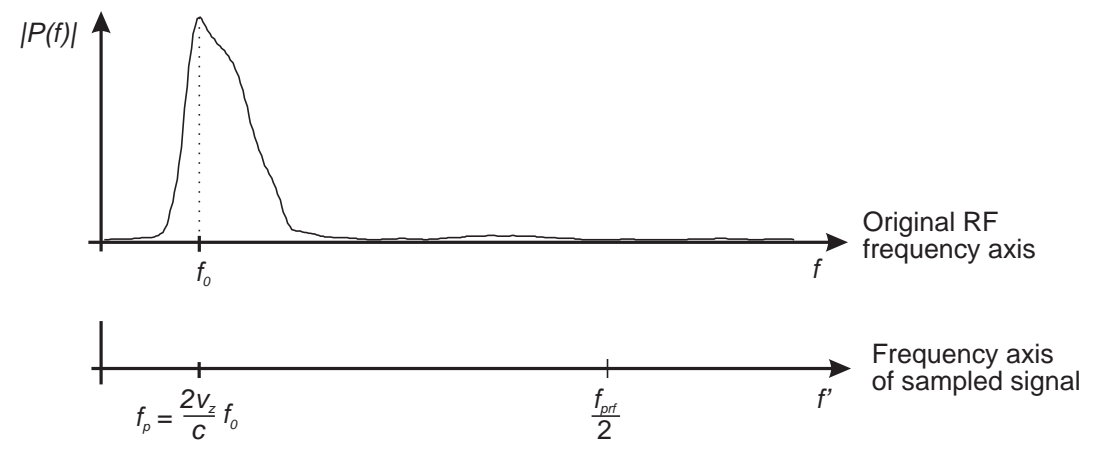

Figure 6: Frequency axis scaling for a sampled signal for a scatterer moving at a velocity of $v_{z}$.

Attenuation will shift the center frequency of the pulse spectrum downwards, but this attenuation downshift is also multiplied with $2 v_{z} / c$. So it is the downshift relative to $f_{0}$ that determines the bias of the estimate.

The instantaneous Doppler shift during the pulse's interaction with the moving scatterer gives a frequency shift of $\frac{2 v_{z}}{c} f_{0}$. This shift is also multiplied with $2 v_{z} / c$, and the resulting frequency deviation is

$$
\Delta f_{d}=\frac{2 v_{z}}{c} \frac{2 v_{z}}{c} f_{0}
$$

An artifact, albeit with a small effect.

Non-linear effects during either propagation or scattering introduces higher harmonics into the spectrum. They alias around $\pm f_{p r f} / 2$ and introduce additional peaks in the spectrum and bias the result. The mean frequency is biased towards higher frequencies, if no aliasing takes place.

\section{DEMODULATION}

The model also makes the role of the demodulation process clear. The main purpose is to generate a signal that can detect the direction of the signal, and to reduce noise in the signal.

The signal after the demodulation can be written as

$$
\begin{aligned}
r_{d}\left(t_{r}\right) & =h_{l p}\left(t_{r}\right) *\left[r\left(t_{r}\right) \exp \left(j 2 \pi f_{0} t_{r}\right)\right] \\
& =r\left(t_{r}\right) *\left[h_{l p}\left(t_{r}\right) \exp \left(j 2 \pi f_{0} t_{r}\right)\right]
\end{aligned}
$$

where $h_{l p}\left(t_{r}\right)$ is a low-pass filter usually implemented as an integration over the pulse duration. It is, thus, possible to use a set of frequency modulated filters, and then apply the sampling operation. For a sinus burst the frequency modulated filter equals the pulse, and is equivalent to a matched filter (Skolnik 1980, Kristoffersen 1986), and can be replaced by such a filter. The demodulation can also be rewritten as

$$
\begin{aligned}
r_{d}\left(t_{r}\right) & =r\left(t_{r}\right) *\left[h_{l p}\left(t_{r}\right)\left(\cos \left(2 \pi f_{0} t_{r}\right)+j \sin \left(2 \pi f_{0} t_{r}\right)\right)\right] \\
& =r\left(t_{r}\right) *\left[h_{l p}\left(t_{r}\right)\left(\cos \left(2 \pi f_{0} t_{r}\right)+j \cos \left(2 \pi f_{0} t_{r}-\frac{\pi}{2}\right)\right)\right] \\
& =r\left(t_{r}\right) *\left[h_{l p}\left(t_{r}\right)\left(\cos \left(2 \pi f_{0} t_{r}\right)+j \cos \left(2 \pi f_{0} t_{r}-\frac{1}{4 f_{0}}\right)\right)\right]
\end{aligned}
$$

The imaginary part of the filter is just a delayed version of the real part. Measuring the signal a quarter period after the real signal, thus, gives a suitable signal (Halberg and Thiele 1986). An alternative is to perform a Hilbert transform on the RF signal to make the $90^{\circ}$ phase shift for all frequencies. 
The low-pass filters in the receiver are matched to the bandwidth of the pulse (Kristoffersen, 1986). This is done to reduce noise, and an alternative demodulation is to do a matched filtration on the received signal, and then a Hilbert transform. A complex sample set is then extracted and processed to yield the spectrum. Further information on direct sampling and its consequences can be found in Jensen (1996).

\section{REFERENCES}

Baker, D. W. Pulsed ultrasonic Doppler blood-flow sensing. IEEE Trans. Son. Ultrason., SU-17:170-185, 1970 .

Forsberg, F., and Jørgensen, M. Ø. Sampling technique for an ultrasound Doppler system. Med. Biol. Eng. Comp., 27:207-210, 1989.

Halberg, L. I., and Thiele, K. E. Extraction of blood flow information using Doppler-shifted ultrasound. HP-Journal, 37:35-40, 1986.

Holland, S. K., Orphanoudakis, S. C., and Jaffe, C. C. Frequency-dependent attenuation effects in pulsed Doppler ultrasound: Experimental results. IEEE Trans. Biomed. Eng., BME-31:626-631, 1984.

Jensen, J. A. Estimation of Blood Velocities Using Ultrasound: A Signal Processing Approach. Cambridge University Press, New York, 1996.

Kristoffersen, K. Optimal receiver filtering in pulsed Doppler ultrasound blood velocity measurements. IEEE Trans. Ultrason., Ferroelec., Freq. Contr., 33:51-58, 1986.

Magnin, P. A. Doppler effect: History and theory. HP-Journal, 37:26-31, 1986.

Newhouse, V. L., and Amir, L. Time dilation and inversion properties and the output spectrum of pulsed Doppler flowmeters. IEEE Trans. Son. Ultrason., 30:174-179, 1983.

Newhouse, V. L., Bendick, P. J., and Varner, L. W. Analysis of transit time effects on Doppler flow measurement. IEEE Trans. Biomed. Eng., BME-23:381-387, 1976.

Newhouse, V. L., Ehrenwald, A. R., and Johnson, G. F. The effect of Rayleigh scattering and frequency dependent absorption on the output spectrum of Doppler blood flowmeters. Ultrasound Med., 3B:1181-1191, 1977b.

Skolnik, M. Introduction to Radar Systems. McGraw-Hill, New York, 1980.

Thomas, N., and Leeman, S. Mean frequency via zero crossings. In Proc. IEEE Ultrason. Symp., pages $1297-1300,1991$.

Thomas, N., and Leeman, S. The double Doppler effect. In Acoust. Sensing and Imag., volume 369, pages $164-168$. IEE, 1993. 